\title{
De la pertinence des variables pour l'étude de la variation
}

\author{
Béatrice Akissi Boutin \\ CLLE - UMR 5263 / Université Toulouse 2 \\ et ILA / Université de Cocody-Abidjan \\ boubeaki@gmail.com
}

\section{Introduction}

La division du matériel linguistique en variétés de langue semble un pré requis de tout discours sur la variation alors qu'une telle classification est loin d'aller de soi. Les variétés «nouvelles » de français en sont une illustration. Parmi les éléments qui varient, peu sont effectivement perçus comme de la variation et encore moins comme une variation significative. En même temps, certains éléments qui ont varié certaines variantes - sont surdimensionnés par rapport à ce qui n'a pas varié en regard à une autre variété. De fait, la perception de la variation est plus ou moins aiguë selon les domaines (phonologique, lexical ou syntaxique) et seules un petit nombre de variantes seront perçues comme des marqueurs de telle ou telle variété (géographique, sociale, situationnelle).

La conscience linguistique des locuteurs se forme à l'aide des stéréotypes qui circulent dans une société, qui eux mêmes vont sans doute de pair avec la normativité de la langue. Ainsi, dans les pays d'Afrique où le français est récemment implanté et où il évolue, dans les deux sens du terme, relativement en marge du français standard, encore peu de signes sont emblématiques d'un style ou d'un groupe social. Dans de tels contextes, il n'est pas certain que le changement soit interprétable avec les mêmes critères que ceux utilisés pour le français en France. Nous proposons l'analyse de quelques variables, phonologiques, lexicales et syntaxiques pour montrer la diversité de leur comportement et la difficulté à les regrouper pour caractériser des variétés de langue. Le texte qui suit ne rend que partiellement compte d'une recherche encore en cours.

\section{Langues, variétés et méthodologie}

Aucun travail scientifique n'a été fait sur les registres de langue en Côte d'Ivoire, ni sur les marqueurs stylistiques, ni même sur les indices linguistiques. Ce sont des classifications opérées sur d'autres variétés géographiques de français qui servent, jusqu'à présent, de points de repère pour les enseignants et les chercheurs. Toutefois, la pertinence de ces critères, pour les sociétés africaines actuelles, n'a pas encore été démontrée.

Nous disposons ici, pour relier des variantes à des variétés de langue, d'une enquête menée à Abidjan sur les représentations et attitudes linguistiques en 1999, des études sociolinguistiques existantes sur le français en Côte d'Ivoire et des premiers résultats de l'analyse d'un corpus de quatorze locuteurs d'Abidjan enregistrés en 2004 et 2005.

\subsection{La perception des variétés de langues par les locuteurs}

L'absence de travaux scientifiques va de pair avec l'absence de symbolique du message vocal (Léon 2005). Il est possible que, dans des régions où l'appropriation de la langue française est encore en cours, beaucoup de signes n'aient pas fait l'objet d'une prise de conscience par la communauté et encore moins ne soient devenus des symboles (conventionnels) d'un groupe.

En Côte d'Ivoire seuls certains traits phoniques, qui fonctionnent comme de simples indices et non comme des signaux, sont reconnus liés à certaines langues africaines. Ces traits ne se transmettent pas seulement dans les milieux où le français est langue seconde, mais aussi dans les milieux urbains où il est 
langue première. Des études détaillées restent à faire, qui puissent isoler les facteurs linguistiques susceptibles d'interférer, sachant qu'il faudra tenir compte, en contexte multilingue, non seulement de la langue première, mais aussi de la langue de l'environnement, de la langue la plus utilisée, des autres langues parlées par le locuteur, etc.

L'enquête que nous avons menée à Abidjan en décembre 1999 peut nous aider à illustrer la difficulté à catégoriser les locuteurs francophones ivoiriens par leur manière de parler. L'enquête s'adressait à des personnes d'une catégorie socioprofessionnelle peu importante en nombre, mais qui pourrait entrer en jeu dans l'objectivation d'une norme locale : enseignants, éditeurs, écrivains, inspecteurs. Ces personnes, dont l'outil de travail est le français, sont portées à évaluer les variétés de langue, à prendre position sur les normes, exogène et endogène et, finalement, jouent un rôle clé dans la circulation et la diffusion des valeurs attachées aux variétés. On pouvait en attendre, du fait de leur profession comme du contexte d'enquête, qu'elles adoptent une position de défense et de diffusion de la norme académique.

Les thèmes de l'enquête qui nous intéressent $\mathrm{ici}^{1}$ sont ceux qui visaient à approcher la perception des variétés de français par ces professionnels de la parole : Comment se représentent-ils les diverses variétés de français? Sont-elles propres à certains groupes de locuteurs différenciés selon le milieu social, le niveau d'instruction scolaire, le lieu géographique (urbain, rural, de telle ou telle région) ? Existe t-il une variation du jugement selon le contexte de communication?

Les témoins ont tous été plus ou moins réticents à cette demande de catégorisation, mais finalement, deux tendances se sont dégagées des réponses : un groupe où les sujets tentent d'établir une division selon des critères sociaux et un groupe qui refuse l'idée d'une classification sociale à partir des usages du français. Les manières dont les personnes interviewées rendent ensuite compte des échanges entre les variétés dépendent de leur attitude face à la catégorisation.

Dans le premier groupe, les sujets opèrent une division en trois groupes selon le niveau d'études : 1 . le « français académique », « des intellectuels », de « ceux qui ont fait de longues études », 2. le « français de la rue », "celui qui est parlé », 3. le français anciennement appelé « de Moussa », des personnes de niveau Primaire ou « qui n'ont pas été à l'école » et qui « ne travaillent pas » (autrement dit, travaillent dans les secteurs informels).

Concernant les échanges entre les variétés, deux professeurs, dont l'un est aussi écrivain, représentent la position la plus sévère et parlent d' « adaptation » des intellectuels à la variété basilectale de français : «Il y a des personnes qui arrivent à parler, enfin à s'adapter au milieu où ils sont, mais sinon, en général ... Bon, les intellectuels pourraient s'adapter un peu plus facilement, alors que les autres, c'est pas évident » (E4). Pour un autre professeur, "si vous êtes universitaire et que vous voulez vous adresser à un paysan vous vous mettez à son niveau. [...] Dans les familles, dans les foyers, dans les ménages, l'homme instruit s'adresse à son domestique dans la langue décousue qui est la sienne. [...] En général, quand je me trouve avec des gens de mon niveau, nous faisons l'effort de parler correctement français » (P4).

Dans le deuxième groupe, les classifications sociales sont rejetées et les témoins opèrent des classifications selon des critères situationnels. Les catégories se confondent alors avec les registres stylistiques de langue : 1. soutenu, 2. « intermédiaire », 3. « relâché », 4. nouchi (argotique) ${ }^{2}$.

Un professeur (P5) fait correspondre, selon le contexte professionnel, des positions différentes sur la langue à donner en modèle et distingue : 1. les anciens instituteurs, qui parlent un « français très châtié », 2. les enseignants, qui « mettent leur point d'honneur à parler un français très correct », 3. les journalistes, dont le français a les mêmes caractéristiques hors de Côte d'Ivoire, 4. certains nouveaux instituteurs, "ils ont changé », dans une situation où « il s'agit même plus de bien parler français ».

La position extrême de ce groupe est représentée par des sujets qui rejettent nettement des distinctions de langue basées sur la catégorie sociale et le niveau professionnel des locuteurs dans les termes suivants : «Il n’y a pas, ici dans ce pays, des barrières étanches entre les catégories sociales, je pense. Il y a de grandes cérémonies ici, qui brassent les différentes couches sociales. C'est, par exemple, les funérailles, les baptêmes, les naissances, etc. Et quand ces gens là se retrouvent, ils ne se retrouvent pas par classes sociales, ils se retrouvent par famille, par relations ; et au niveau des relations il n'y a pas cette coupure- 
là. » (I2). Et : « Non peut-être pas à ce niveau, pas les classes sociales. Vous savez, même l'intellectuel ivoirien, quand il redescend dans son milieu naturel, il rejoint ce français, moi je le ferais dans ma famille » (E3).

Dans l'ensemble, les sujets enquêtés perçoivent l'existence de plusieurs variétés de français se spécifiant selon le contexte de communication, mais aussi l'existence d'attitudes linguistiques se différenciant selon les générations. Cependant, l'hétérogénéité des représentations des variétés de langue montre toute la difficulté de les classifier selon des critères sociaux. La catégorisation selon le niveau d'études est significative du statut du français en Côte d'Ivoire : il reste langue étrangère dans sa variété académique ou acrolectale, même s'il est langue seconde ou première selon les cas et les familles, dans ses variétés mésolectale et basilectale.

Ce qui rend difficile la catégorisation des locuteurs ivoiriens est que les traits spécifiquement ivoiriens, appelés parfois « français populaire ivoirien », font partie de la compétence linguistique quels que soient le niveau d'études et la profession exercée. Tous les francophones de Côte d'Ivoire, y compris les résidents et les locuteurs les plus opposés à la norme locale de français ou au «français ivoirien » usent eux aussi, peut-être à leur insu, de ces structures spécifiques.

\subsection{Les classifications des chercheurs}

Gadet 2007 discute la notion de variétés de langue et montre qu'une variété est avant tout une construction homogénéisante, la «représentation du groupement d'usages variables d'un groupe, reflétant plus ou moins les usages reconnus par les membres de la communauté. (Gadet 2007 : 177) ». Nous pouvons cependant accepter ici cette notion tant qu'elle n'occulte pas la dynamique de la variation (Gadet $2007: 26$ ).

Depuis quelques années et sans doute dans un souci de conformité aux découpages classiques, les chercheurs divisent le français en Côte d'Ivoire, en quatre grands types d'usages ou variétés, la première régie par la norme exogène et les trois autres par une norme endogène qui ne cesse de gagner du terrain :

Le français académique, proche du français standard de France, est le français officiel, mais ne s'entend que dans les établissements scolaires et universitaires, le secteur économique formel et les échanges internationaux. Le français courant ivoirien est issu d'un rapprochement des variétés basilectales et mésolectales durant les dernières décennies du $\mathrm{XX}^{\mathrm{e}}$ siècle. Le français populaire ivoirien, héritier du français véhiculaire diffusé sur le territoire de la colonie au début du $\mathrm{XX}^{\mathrm{e}}$ siècle et du français populaire d'Abidjan (Hattiger 1981; Ploog 2002), est omniprésent dans la rue et les foyers. Il est la seule langue véhiculaire neutre, les autres (dioula, agni-baoulé) étant encore marquées ethniquement dans les représentations collectives. La quatrième variété de français à occuper le terrain linguistique est le nouchi, argot jeune fortement imprégné des langues et réalités locales. Il affiche un dynamisme extraordinaire depuis la fin des années quatre-vingt (Kouadio N'Guessan 1992 et 2005).

Cette classification est, cependant, contestée pour plusieurs raisons. Brou-Diallo 2004 montre, par une étude sur la perception des énoncés, que les limites entre les variétés sont de moins en moins perceptibles. Abolou 2007 remet en cause le dénommé «français populaire d'Abidjan », "français populaire ivoirien », « français de Moussa » ou « abidjanais » dont le repérage est soit diastratique, soit diatopique, soit diaphasique, selon les chercheurs et les corpus constitués.

Les travaux de Knutsen (2007 a et b) vérifie statistiquement sur un corpus recueilli en 1998-1999 qu'il n'existe pas en Côte d'Ivoire de variétés de français étanches et que seule la fréquence d'apparition de traits «non-standard» varie selon les locuteurs. Après avoir mis en correspondance trois variables extralinguistiques : l'attitude face à la norme locale, le niveau scolaire et le style formel ou informel du discours, et neuf variables syntaxiques, elle conclue que les locuteurs scolarisés après le BEPC constituent le groupe le moins homogène quant au comportement linguistique. Leurs usages dépendent de leur attitude (positive ou négative) face à la norme locale, elle-même liée à l'ambition (et à la possibilité réelle) de s'insérer professionnellement dans le secteur formel. 
Knutsen 2007 donne cependant une vision trop restrictive de l'attitude face au « français ivoirien », sans doute du fait du petit nombre de témoins adultes ayant fait des études supérieures. Comme l'avait montré notre enquête de la même année, la norme exogène est valorisée dans les limites du contexte académique, du secteur formel et des échanges internationaux. Elle est dépréciée hors de ces situations, jusque par les tenants de la norme académique. Dans tous les autres contextes, c'est la norme endogène qui est appréciée, à des degrés divers ; elle bénéficie de l'appui d'attitudes identitaires fortes qui en assurent une représentation unifiée.

\subsection{Corpus et méthode de l'enquête de 2004-2005}

L'étude qui suit se base sur une enquête menée à Abidjan en 2004-2005 dans le cadre du projet PFC ( Phonologie du français contemporain. Usages, variétés et structure ») ${ }^{3}$ auprès de 14 locuteurs résidant dans la capitale économique depuis de nombreuses années. Les locuteurs ont été choisis selon les paramètres sociolinguistiques d'âge, sexe, niveau d'études et groupe ethnique; tous parlent habituellement en français dans leur travail, leur foyer et dans la rue. Nous avons réalisé cette enquête avec l'appui de l'Institut de Linguistique Appliquée, Université Cocody-Abidjan. Jean-Martial Kouamé, alors doctorant, a participé comme enquêteur non connu des témoins. Le réseau utilisé (Milroy 1980) a été celui de l'école professionnelle Yarani, située à l'entrée du quartier populaire d'Abobo, où passent une grande quantité de personnes, issues de plusieurs milieux d'Abidjan : élèves recrutés avec le niveau $5^{\text {ème }}$ à $3^{\text {ème }}$ en formation de CAP ou de BT ; professionnels en formation continue ; adultes du quartier en formation du soir ; enseignants et animateurs divers. Les témoins sont des proches et amis de l'enquêtrice, ou sont introduits par ceux-ci : conjoints, parents, professeurs, élèves, etc.

Chaque témoin est enregistré 50 à 60 minutes, pendant qu'il réalise quatre activités : une lecture de mots, une lecture de texte, un entretien formel guidé par l'enquêteur non connu et une conversation libre et informelle avec l'enquêtrice proche. Actuellement sont transcrites sous Praat 76 minutes d'entretiens guidés et 97 minutes d'entretiens libres. Notre corpus est complémentaire d'autres corpus oraux existants pour le français en Côte d'Ivoire, dans le sens qu'il recueille la parole de témoins de niveaux d'études et professionnels divers, dont certains relativement plus hauts que celui de ces corpus. D'une part, les tâches de lecture constituent une première sélection des locuteurs, d'autre part, la moitié des locuteurs exerce une profession ou occupation stable.

Le corpus a initialement un but phonologique ; il peut toutefois être utilisée à d'autres fins (études lexicales, syntaxiques, énonciatives, etc.). D'un point de vue sociolinguistique, il donne accès à des études qualitatives, l'entretien guidé permettant de dresser finement le profil sociolinguistique du locuteur. Ainsi, bien qu'aucune question n'ait porté sur l'attitude face à la langue, il nous est possible de dire que la plupart des témoins de l'enquête n'ont pas une attitude négative face à l'ivoirisation du français. En revanche, certains d'entre eux déplorent soit l'omniprésence du français populaire, soit quelques traits stigmatisés, soit le manque de compétence parallèle des locuteurs dans un français plus international.

L'absence d'études sociolinguistiques (à part Knutsen 2007 a et b) sur les variables pertinentes pour le français en Afrique empêche toute prédictibilité. L'enquête permet cependant de vérifier la différence de style entre lectures, conversations formelles et conversations informelles, la pertinence de certaines variables selon des facteurs diastratiques et diaphasiques, ainsi que la non pertinence de certaines autres variables, de ces mêmes points de vue.

\section{Les variables phonologiques}

Plusieurs unités linguistiques, du segmental au supra segmental sont analysées par Léon 2005 comme des indices sémiotiques de style. Selon ce même auteur, liaison facultative et «e caduc» font partie des unités linguistiques qui peuvent être interprétées à des fins "phonostylistiques». Une liaison interdite réalisée, par exemple, implique une "indexation sémiotique ». Le " e caduc », en plus d'être indice de dialecte, est, dans une moindre mesure, indice de style en français du Nord de la France : peu de «e 
caduc » et de liaisons facultatives accompagnent un style «familier », beaucoup de « e caduc » et de liaisons facultatives accompagnent un style « recherché ». Cependant, ces analyses sont à nuancer avec celles qui ont été réalisées sur le corpus PFC de France métropolitaine, comme, par exemple, Durand \& Lyche 2008. Leurs résultats montrent, d'une part, qu'on observe peu de variation pour le schwa et à peine plus pour la liaison et, d'autre part, que la variation est un phénomène complexe pour lequel interviennent de nombreux facteurs systémiques, énonciatifs et pas seulement stylistiques.

Dans le but de rechercher quelles sont, parmi les variantes présentes dans le corpus PFC - Côte d'Ivoire, celles qui constituent des indices significatifs, nous analysons tour à tour plusieurs variables, dont les variantes sont, en français hexagonal, souvent considérés comme des marqueurs stylistiques: les réalisations du $\mathrm{R}$, les liaisons, les schwas et le coup de glotte en initiale vocalique.

\subsection{Les réalisations du $\mathbf{R}$}

L'analyse des tâches de lecture de notre corpus met en évidence une grande variation des réalisations du $\mathrm{R}$. Le R simple est réduit en finale, en position intervocalique et même en initiale, et ce dans la liste de mot lue, qui mobilise a priori chez le locuteur le plus de vigilance sur sa prononciation. Le seul extrait du relevé des $\mathrm{R}$ initiaux montre, en position forte, tout l'éventail des dix réalisations possibles dans la langue (Boutin \& Turcsan 2006).

La large gamme de réalisations du $\mathrm{R}$ recouvre deux zones d'articulation : la zone alvéolaire et une large zone postérieure allant de la zone vélaire à la zone glottale. Les locuteurs se distribuent, à part égales dans notre corpus, trois patrons de réalisations et affaiblissement du $\mathrm{R}$ :

1) Chez un premier groupe de locuteurs, le R est toujours réalisé de la zone postérieure à la zone glottale, avec plus ou moins de friction $([\mathrm{\zeta}]$ et $[\chi] /[]$ et $[\hbar]>[\gamma]$ ), ou aucune friction (pour $[\mathrm{h}]$ ), ou avec l'approximante vélaire [u] qui revient à une vocalisation de la consonne.

2) Chez un deuxième groupe de locuteurs, le $R$ est toujours réalisé dans la zone alvéolaire, avec des vibrantes alvéolaires $[\mathrm{r}$ ] plus ou moins battus et l'approximante correspondante $[\mathrm{I}]$.

3) Chez un troisième groupe de locuteurs, le R est réalisé prioritairement dans la zone postérieure, mais souvent aussi dans la zone alvéolaire. Ces locuteurs possèdent tout l'éventail des réalisations possibles du $\mathrm{R}$ en Côte d'Ivoire et tous les schémas d'affaiblissement de la consonne.

Pour ces trois groupes de locuteurs, une corrélation nette entre le paradigme des réalisations du $\mathrm{R}$ et des facteurs diastratiques tels que l'âge, la langue première, le niveau d'études, le niveau socioéconomique, est impossible à déterminer et l'on est forcé de conclure à une variation libre du $\mathrm{R}$ dans le contexte actuel. De nombreux autres facteurs sociolinguistiques interviennent probablement, difficiles à cerner, tels que l'habitat de l'enfance (rural ou urbain), la profession, la période de scolarisation, l'origine (africaine ou européenne) des instituteurs ... A ces paramètres se joignent les représentations sociales et les jugements épilinguistiques : alors que le [r] est la version traditionnelle du R, marquée, exemplaire, utilisée par les enseignants des premières générations ou pour épeler un mot, la prononciation typiquement ivoirienne urbaine est une version faible, articulée dans une zone postérieure étendue et floue avec le minimum ou pas du tout de friction. La fricative uvulaire forte $[в]$ et sa version sourde $[\chi]$, quant à elle, caractérise « l'accent français » ou « chôcô ».

Tous ces facteurs peuvent expliquer le large éventail de réalisations du $\mathrm{R}$ mais ne peuvent en aucun cas les prédire : malgré la richesse du phénomène, la variation du $\mathrm{R}$ ne peut constituer une variable pertinente pour observer la variation diastratique du français en Côte d'Ivoire. 


\subsection{Les liaisons}

L'état de la liaison en français a récemment été dressé par Durand \& Lyche 2008. Peu d'originalité caractérise notre corpus dans ce domaine et la valeur diaphasique et diastratique des variables concernant les liaisons sont similaires.

Aucune liaison interdite n'existe dans le corpus d'entretiens. Les liaisons obligatoires en [n] (en, on, un), en $[z]$ (clitiques de personnes et déterminants pluriels), après quelques, tout sont toutes faites à la lecture comme en conversation.

La liaison facultative après est est variable, mais cette variabilité ne dépend pas du contexte de conversation ou de lecture : $50 \%$ des liaisons sont réalisées en ce lieu à la lecture comme en conversation.

Les lieux de variation selon le style sont les liaisons obligatoires après les prépositions dans, chez et les liaisons facultatives après un nom pluriel. Alors que les liaisons après une préposition sont toutes réalisées à la lecture, elles sont susceptibles de ne pas l'être (quoique très rarement) en contexte de parole spontanée. L'adverbe très a toujours été suivi d'une consonne de liaison en contexte prévocalique à la lecture, alors que la liaison n'est pas toujours réalisée en oral spontané. D'autre part, alors qu'aucun nom pluriel suivi d'un adjectif à initiale vocalique n'est lieu de liaison en contexte de parole spontanée, ils peuvent l'être à la lecture par les locuteurs de plus hauts niveaux d'études (11\% de liaisons réalisées dans ce contexte).

Seules les liaisons après les prépositions et l'adverbe monosyllabiques (dans, chez, très) et celles après un nom pluriel constituent des variables permettant de distinguer la lecture de la parole spontanée. A l'oral, les occurrences de dans, chez, très ne sont pas suffisantes dans le corpus pour différencier l'oral formel de l'oral surveillé.

\subsection{Les schwas}

Le comportement du schwa présente plus d'originalité que celui des liaisons par rapport à d'autres variétés géographiques de français. On peut se demander si un schwa existe réellement en français de Côte d'Ivoire, dans le sens d'une voyelle dénotée par un -e- graphique qui alterne avec zéro. Les comptages réalisés permettent en effet de confirmer ce que la pratique de la variété laissait entrevoir.

- Dans les monosyllabes et en début de mots, les schwas sont réalisés à 95,5\% et $97 \%$ respectivement. Dans les suites de monosyllabes (par exemple il faudrait que je le suive), le schwa est conservé dans les mêmes proportions.

- En finale de mots, les schwas ne sont pas conservés à $97 \%$ : $2 \%$ seulement sont réalisés en conversation, $9 \%$ sont réalisés dans la lecture du texte. Ce phénomène est en étroite dépendance de la chute des consonnes finales.

- Les syllabes internes font apparaître le seul vrai schwa : $35 \%$ sont réalisées dans cette position et on observe réellement une variation entre les locuteurs et chez le même locuteur. Toutefois, les proportions de schwas réalisés en situation d'oral spontané ou de lecture sont inverses : alors qu'à la lecture, $63 \%$ de schwas internes sont réalisés, en conversation, $26 \%$ sont réalisés. Par ailleurs, le caractère formel / informel de l'entretien n'influe pas sur la réalisation / non réalisation des schwas.

Si davantage de schwas sont prononcés à la lecture, en finale de mots et surtout en syllabes internes, on ne peut pour autant conclure que la réalisation du schwa fasse partie de la norme telle qu'elle est intériorisée par les locuteurs. Le phénomène peut être imputé au processus de lecture qui fait que le locuteur voit le e - graphique, ou encore à l'effet d'une articulation soignée des consonnes. Ainsi, le pourcentage plus élevé de schwas finaux dans la lecture du texte peut s'expliquer par l'effort des locuteurs de prononcer les consonnes finales.

Tout comme la réalisation du $\mathrm{R}$, la présence $v s$ absence de schwa en syllabe interne ne peut pas non plus être corrélée à des facteurs sociaux. Du fait que la prononciation du schwa dans cette position est associé 
à une situation de lecture qui rappelle le contexte scolaire et la norme académique, on doit reconnaître que celle-ci s'éloigne sur ce point de la norme hexagonale.

\subsection{Le coup de glotte}

En français hexagonal, le coup de glotte [?] n'est pas un phonème et fonctionne comme indice de colère ou comme signal d'injonction ou d'insistance. Il peut aussi être marqueur d'un style professionnel pour caractériser, par exemple, la presse oralisée (Léon 2005).

En français de Côte d'Ivoire, le coup de glotte est réputé plus fréquent que l'enchaînement lorsque deux mots sont séparés par deux voyelles. Il est perçu comme un indice «d'accent africain » en général. Il n'existe encore aucunes données chiffrées d'une comparaison entre des variétés "européennes » et « africaines » de français, mais notre corpus ivoirien montre, qu'en contexte de liaison potentielle, $25 \%$ des liaisons non réalisées sont le lieu d'un coup de glotte. Les résultats selon l'activité font apparaître une opposition nette entre lecture et conversation : $75 \%$ pour la lecture et $12 \%$ pour les conversations. De plus, les deux styles de conversation sont départagés eux aussi par cette variable, puisque les coups de glotte augmentent légèrement en entretien formel : $15 \%$, contre $10 \%$ en entretien informel. Dans notre corpus, le coup de glotte apparait donc, sans aucune valeur émotive, comme un marqueur de clarté et de formalisme de l'énonciation.

\section{Les variantes syntaxiques}

En soi, le comptage des phénomènes syntaxiques n'apparaît pas aussi probant que celui des faits phonologiques, puisque, dans le cas de la phonologie, les occurrences d'un allophone peuvent être comparées aux occurrences d'un autre allophone alors que dans celui de la syntaxe, les formes à comparer ne sont pas aussi facilement repérables. Les formes à mettre en correspondance doivent avoir le même fonctionnement dans le système, c'est-à-dire être syntaxiquement équivalentes mais aussi avoir le même sens. Or, le lexique, l'ordre des mots, mais aussi le contexte de communication interfèrent souvent pour créer des différences de sens entre diverses phrases qui pourraient être prises comme des variantes, mais qui ne sont plus sémantiquement équivalentes.

De plus, il est impossible de comparer quantitativement des formes existantes par rapport à celles qui auraient pu être réalisées, comme il est impossible de prédire l'apparition d'une construction plutôt qu'une autre. Les stratégies d'évitement d'une forme comportent un tel éloignement de celle-ci qu'il est souvent peu probable de les interpréter comme de telles stratégies. Par exemple, une construction hypothétique concurrence le conditionnel en français ivoirien, formée avec aller à l'imparfait, dans des phrases comme :

(1) Je dis, c'est, c'est, c'est Dieu qui a fait, parce que tels qu'ils ont été surpris, en principe, on n'allait pas pouvoir, ils n'al/, ils n'auraient pas pu se, se, se, se libérer. (CIAIEL)

Dans un contexte de langue formelle ou académique, un locuteur peut très bien bannir de son usage les formes en aller sans pour autant que les formes au conditionnel augmentent (ou apparaissent) dans son discours - ce qui n'est pas le cas de la locutrice de (1).

Le concept de variables est donc difficile en syntaxe, du moins appliqué à notre petit corpus. Des variantes par rapport au français (standard) de France sont, en revanche facilement repérables. Les travaux déjà réalisés sur le français de Côte d'Ivoire montrent que l'usage soutenu de la langue y est proche d'un usage équivalent en France, alors que l'usage «populaire » s'éloigne davantage d'un usage équivalent en France. Nous avons testé, d'une part l'éloignement $v s$ proximité du français hexagonal en conversation par la quantité de constructions spécifiquement ivoiriennes et, d'autre part, l'éloignement $v s$ proximité du français standard « soutenu » par la quantité de constructions particulièrement soignées. 


\subsection{Les spécificités syntaxiques ivoiriennes}

Le repérage des variantes qui puissent être quantifiées exclut une analyse fine des structures syntaxiques. Nous nous sommes reportée pour le choix des constructions propres au français ivoirien, aux descriptions existantes (Kouadio 1999, Ploog 2002, Boutin 2002, Knutsen 2007). Nous n'avons pas retenu les constructions non standard qui peuvent être observées dans les variétés hexagonales de français. Par exemple, ne posent aucun problème des relatives dites populaires en que, répandues dans toutes les variétés de français, du type :

(2) Ensemble, nous devons lutter aussi contre plein de choses. Il y a plein de choses que nous devons lutter, la corruption, euh. (CIASBL)

Le là postposé à un syntagme nominal introduit par un déterminant démonstratif n'est pas retenu comme une spécificité ivoirienne :

(3) Et justement, dans ces produits chinois là, vous avez des produits à base de plantes. (CIASFL)

Au contraire, le là postposé à un syntagme nominal défini est relevé, tout comme le là postposé à un autre syntagme (adverbial, verbal). Dans cette position, le là est perçu comme spécifiquement africain dans la littérature scientifique, mais il est probable qu'il puisse être observé aussi dans des variétés européennes de français (Voir Boutin 2007,c).

(4) Le sang de la chèvre là, elle fait, griller ça, c'est très bon.

(5) Donc, quand je commence là, elle s'émotionne. (CIAAKL)

D’autres variantes ont été appréciées, comme :

- dans la syntaxe du nom :

- ça/c' en reprise d'un syntagme nominal défini (Voir Knutsen 2007) dans des phrases comme :

(6) Donc, le gombo i/, il y a eu une dispute. Donc, le gombo a poussé la toma/, euh, l'aubergine, elle est tombée sur ses fesses. Donc c'est resté plat. C'est pour ça l'aubergine est plat. (CIAAKL)

- 1'absence de déterminant (Voir Boutin 2007,a), comme dans :

(7) On mange sauce graine, sauce arachide, sauce aubergine. On mange plus sauce gombo. (CIAEO1L)

- le déterminant le/les introduisant un syntagme nominal nouveau (Voir Knutsen 2007)

(8) Elle fait des dessins. Ca dépend des mo/, ça dépend des motifs. Elle peut faire des trucs, genre les jumelles, les jumeaux. (CIAAKL)

- l'absence d'accord en genre dans le syntagme nominal, comme dans (6), plat,

- un introduisant un syntagme nominal complément d'un verbe négatif

(9) Tu vas à l'école, tu finis, aujourd'hui tu es là, tu, tu es encore, tu es là, tu traînes. Tu n'as pas un b/boulot. (CIASBL)

- dans la syntaxe du verbe :

- l'omission du complément direct ou indirect (Voir Boutin 2005),

(10) Chacun cotise, pour pouvoir avoir une somme plus, euh, plus élevée par rapport aux autres, pour pouvoir montrer. On appelle ça la démonstra, quoi.

- l'indicatif à la place du subjonctif, comme dans :

(11) Ils veulent que, bon, tu t'occupes bien de la fille $\mathrm{Ce}<\mathrm{E} 1$ : Ah, oui. $>$ qui est sûr, qu'il y a l'entente. (CIAAKL)

- l'absence du futur dans le passé ou de l'imparfait demandés par la concordance des temps, comme : 
- l'absence du subordonnant complétif que (Voir Boutin 2007,b), comme dans la phrase précédente,

- l'absence du subordonnant relatif que,

- un changement de préposition locative (Voir Boutin 2006),

- le changement entre une construction verbale directe et prépositionnelle,

- dans la syntaxe textuelle :

- l'absence de concordance des pronoms dans une même unité syntaxique, comme, par exemple :

(13) Oui, ils donnent quelque chose. On donne ce que tu as. (CIAAKL)

- la construction non subordonnée en pour suivi d'un verbe à l'infinitif(Voir Boutin 2007,b),

- que introducteur de paroles rapportées au « discours direct » (Voir Prignitz 2006)

(14) Il va à cette dame pour lui dire que bon, c'est pour ton, euh, ton petit café. (CIASBL)

- le redoublement, comme dans :

(15) Elle crie crie trop trop sur les gens. (CIAEO1L)

(16) Par exemple, on fait les couleurs deux, deux, on choisit deux couleurs, on dit 'Bon, euh, rouge et, euh, bleu, vous allez travailler. (CIASNL)

(F : Par exemple, on fait les couleurs deux par deux, on choisit deux couleurs...)

Les résultats de l'analyse des conversations formelles et informelles confirment la différence de style de ces deux situations. Le total des variantes syntaxiques enregistrées pour l'ensemble des entretiens informels est deux fois et demie plus élevé que celui des entretiens formels. Les locuteurs qui ont le plus de spécificités ivoiriennes sont trois jeunes, de niveau d'études moins avancé et un médecin. Tous les locuteurs montrent une différence de style entre les deux conversations, mais cette différence peut être plus ou moins prononcée. Sept locuteurs, jeunes ou âgés mais ayant tous (sauf une) fait des études supérieures, ne manifestent aucune spécificité en entretien guidé. Parmi eux, certains gardent plus ou moins la même attitude en entretien informel, alors que d'autres sont parmi les locuteurs qui affichent le plus de «syntaxe ivoirienne » (jusqu'à quatre fois plus de spécificités ivoiriennes en style informel). Toutefois les locuteurs qui marquent la plus grande différence entre les deux styles ne sont pas homogènes. Il semble que la différence de style entre les deux conversations traduit une attitude face à la langue qui n'est pas déterminée par le niveau d'étude, la profession ou l'âge.

Parmi les dix-sept variantes répertoriées, six sont les plus fréquentes et représentent $63 \%$ des variantes totales. Ce sont, en ordre décroissant : l'omission du complément verbal; le là postposé ; le déterminant défini pour un nom nouveau; l'absence de concordance de temps; l'omission du subordonnant que; l'absence de concordance de pronom. Il est impossible, à ce stade de l'analyse, d'observer une échelle implicationnelle entre ces variantes.

La comparaison des deux registres met en évidence que les locuteurs ont conscience des formules à bannir de leur discours en contexte formel et on peut conclure à une inhibition des traits syntaxiques ivoiriens dans le style formel. Le français ivoirien apparaît comme le français spontané, de plus en plus marqué des spécificités ivoiriennes à mesure qu'il s'éloigne de la pression de la norme académique et qu'il est moins surveillé. Il reste à vérifier si le style formel fait apparaître davantage de tournure soignées et recherchées de pair avec le bannissement des traits « ivoiriens ».

\subsection{Le soin de la langue standard}

Notre recherche des marqueurs de français recherché, ou d'un soin particulier de la langue part de l'hypothèse que tout locuteur possède des tournures plus sophistiquées qu'il maîtrise et peut insérer dans son discours. Nous avons testé quelques structures représentées dans le corpus : 
- dans la syntaxe du verbe :

- la présence de la particule préverbale ne de négation,

- les subjonctifs et imparfaits employés du fait d'une contrainte syntaxique ou d'une concordance avec le verbe principal, comme dans (17). Seuls les subjonctifs marqués ont été pris en compte, et non les formes non marquées pouvant être interprétées comme des subjonctifs mais aussi comme des indicatifs.

- le conditionnel utilisé dans des structures à valeur hypothétique,

- les gérondifs et participes présents,

- le subordonnant que utilisé pour introduire une deuxième subordonnée coordonnée, comme dans :

(17) Quand il me parlait en français, que je, je savais de quoi il s'agissait, je, je lui répondais en français. (CIAAKG)

- dans la syntaxe du nom :

- des relatives qu'on peut qualifier de «soutenues » : relatives appositives qui n'interviennent pas dans l'identification du référent de l'antécédent et relatives introduites par un relatif composé, du type de :

(18) Je parle le tagbana, qui fait partie des, du groupe sénoufo. (CIAAKG)

(19) Là où j'avais un oncle chez qui je pouvais au moins habiter. (CIASNG)

La comparaison de la fréquence de deux variantes dans les deux styles est faussée par le genre de la conversation. Ainsi, le plus grand nombre de conditionnels et de particules préverbales ne se trouve dans les entretiens libres (le double de ne et $30 \%$ de plus de conditionnels en entretien libre), ce qui ne signifie pas que ces variantes doivent être prises comme des marqueurs d'un style informel, mais plutôt d'un genre conversationnel (Gadet 2000). En effet, en entretien formel, le locuteur doit répondre à des questions sur lui-même et sur ses proches (langues parlées, études faites, occupations, etc.), ce qui rend, effectivement, peu probables les constructions négatives et hypothétiques.

Les résultats de l'analyse de la fréquence des autres variantes montrent que seulement trois constructions « soutenues» apparaissent beaucoup plus souvent dans les entretiens formels. Nous avons ainsi, pour chaque variable, les augmentations suivantes : 1) formes en -ant : $280 \%, 2$ ) relatives soutenues : $170 \%$, 3) subjonctifs et imparfaits : $130 \%$. Le que en deuxième subordonnant est stable dans les deux styles.

Si l'on ne considère que le total des trois traits les plus pertinents du style soutenu, l'attitude face au français ivoirien est corrélée à un comportement inverse en ce qui concerne le français standard chez la moitié des locuteurs. Les locuteurs qui suivent cette tendance se répartissent en deux groupes : ceux qui allient faible fréquence de traits syntaxiques «ivoiriens» et usage supérieur à la moyenne des traits « soutenus », et ceux qui allient forte fréquence de traits syntaxiques ivoiriens et usage inférieur à la moyenne des traits «soutenus ». L'autre moitié des locuteurs compose son discours en entrelaçant toutes sortes de traits, ce qui empêche de mettre vraiment en opposition français ivoirien et français standard. Les résultats comparés de 4.1 et 4.2 vont, à notre avis, dans le sens d'une valorisation des traits du français ivoirien.

\section{La variation lexicale}

Le repérage des mots ivoiriens s'est fait par leur forme et leur sens, le changement de sens étant parfois l'effet d'un changement de construction. Ainsi, des mots spécifiquement ivoiriens par leur forme même sont, par exemple, des interjections n'existant pas en français hexagonal comme tchê (probablement emprunté au dioula: "homme»), des clics, des mots empruntés au nouchi, comme : béou, yèrè. L'existence de mots dont la forme existe en français hexagonal, avec un sens différent, est illustrée par ethnie (pour « langue », on parle aussi notre ethnie), langue (pour « langue africaine », On communiquait en langues. Je parle aucune langue.), rester pour « habiter». Des exemples de mots dont le sens change $\mathrm{du}$ fait d'une utilisation syntaxique différente de celle du français hexagonal sont: déloger et redéployés dans: 
(20) La guerre nous a fait déloger. Depuis le mois de novembre deux mille trois, on a demandé le redéploiement de l'administration. Et mon mari faisant partie du, du corps préfectoral, c'était les premiers à, partir sur le terrain. Euh, donc je, euh, étant fonctionnaire, à la DAF, donc, je n'ai pas été redéployée pendant ce temps. Il y a certains qui ont été redéployés. (CIASNG)

Le comptage des mots spécifiques ivoiriens donne 43 mots types. Les deux registres d'entretiens, guidé et libre, ne se différencient pas par le nombre total de mots types, mais les locuteurs répartissent différemment les mots ivoiriens dans les entretiens de type guidés et libres. Trois locuteurs (d'âge et de conditions sociales diverses) n'en n'utilisent aucun, certains en utilisent le même nombre dans les deux types d'entretien, d'autres les privilégient dans un type d'entretien, formel ou informel, qui varie selon les locuteurs. Autrement dit, cette variable n'est d'aucune pertinence pour différencier entretien formel et informel, âge, niveaux d'études et niveaux professionnels.

Ce qu'on peut tirer du relevé des items ivoiriens est qu'ils reflètent le thème de la conversation, comme on pouvait s'y attendre. Dans les entretiens guidés, les questions sur les langues font apparaître prioritairement les mots ethnie, langue, se débrouiller, les questions sur le temps passé dans les divers lieux de vie, les mots : faire (dans le sens de passer, dans, par exemple : j'ai fait deux ans à Bouaké.), durer, rester, sur la scolarité : fréquenter. Plusieurs interprétations de ces résultats sont possibles. Il se peut qu'aucune conscience linguistique n'accompagne l'utilisation des mots spécifiquement locaux, mais seule la comparaison de nos entretiens avec des entretiens similaires faisant intervenir des locuteurs ivoiriens et des étrangers francophones pourrait le confirmer. Il est plus probable que la représentation d'un français formel en contexte ivoirien ne bannisse pas les items lexicaux spécifiquement ivoiriens. Dans ce cas, ces mots ne constituent pas des marqueurs stylistiques, contrairement à ce que nous avons vu pour les structures syntaxiques « ivoiriennes ».

\section{Conclusion}

Beaucoup de questions ont été soulevées mais le travail de repérage des variables linguistiques à corréler à des variables sociales et stylistiques est loin d'être achevé. Peu de variables ont jusqu'à présent été testées en fonction de facteurs diastratiques, diaphasiques et diachroniques, mais notre étude, à ce niveau, conforte déjà plusieurs notions, qui constituent une base de travail pour la suite.

Les variables n'ont pas toutes le même comportement et ne peuvent pas toutes être analysées avec la même méthode. Des allophones peuvent être facilement repérées en phonologie, mais le concept de variables est moins opératoire pour le lexique et surtout en syntaxe. Un travail d'étiquetage syntaxique sur un corpus plus vaste est nécessaire pour parvenir à des résultats plus solides.

La variation est interne à la langue et n'est pas toujours significative. Toute variante n'est pas un marqueur social et toutes les unités linguistiques n'ont pas autant d'incidence sur la perception d'un style d'interaction. Ainsi pour l'instant, mis à part le R standard, les autres allophones, prononciation antérieure ou postérieure faible du R, sont neutres. Certains marqueurs stylistiques ont la même valeur en France et en Côte d'Ivoire, comme la liaison, alors que d'autres, comme le coup de glotte, sont totalement indépendants. Lorsque la fréquence d'un phénomène est inverse en style formel en France et en Côte d'Ivoire, une opposition entre norme endogène et norme exogène apparaît clairement, c'est le cas des schwas internes. De même, lorsque le locuteur insère autant de mots spécifiques ivoiriens dans ses discours formel et informel, il manifeste ainsi que la norme locale a entériné ces mots, confirmant son éloignement du standard.

Le locuteur, qui manie la diversité de la langue à sa convenance, fait des choix qui n'en excluent pas d'autres. Ainsi, la présence de structures syntaxiques « ivoiriennes » se mêle dans la majorité des discours avec des structures « soutenues ». Mais, si la présence d'éléments qui appartiennent à une variété n'exclut pas ceux qui appartiennent à une autre variété, le concept même de variété comme un tout homogène et singulier est remis en cause. 


\section{Références bibliographiques}

Abolou, C. R. (2007). De l'apport de l'information statistique à la constitution des corpus de français poulaires africains : cas du français populaire d'Abidjan. Revue du Réseau des Observatoires du Français Contemporain en Afrique $\mathrm{n}^{\circ} 22$. Paris : Didier - Erudition, pp.107-126.

Brou-Diallo, A. C. (2004). Aspects des difficultés d'apprentissage du français langue étrangère par des étudiants anglophones africains. Thèse de doctorat, Université de Montpellier III.

Boutin, B. A. (2002) : Description de la variation: Etudes transformationnelles des phrases du français de Côte d'Ivoire, Villeneuve sur Ascq : Presses Universitaires du Septentrion.

Boutin, B. A. (2003) : «Des attitudes envers le français en Afrique : Enquête au sein de professions dont l'outil est le français en Côte d'Ivoire ", Education et Sociétés Plurilingues n¹4-juin : 69-84, Aosta, Italie.

Boutin, B. A. (2005) : «La variation dans la construction verbale en français de Côte d'Ivoire », Revue Québécoise de Linguistique, $\mathrm{n}^{\circ} 32.2$ : 15-45, Montréal.

Boutin, B. A. (2006) : «Adpositions locatives en français de Côte d'Ivoire, en dioula et en baoulé », CORELA vol. 4, $\mathrm{n}^{\circ}$ 1, http://edel.univ-poitiers.fr/corela/document.php?id=1152.

Boutin, B. A. (2007)a : « Déterminant zéro ou omission du déterminant en français de Côte d'Ivoire », Le français en Afrique, Revue du Réseau des Observatoires du Français Contemporain en Afrique Noire ${ }^{\circ} 22: 161-182$. Nice : I.L.F. / CNRS-UMR 6039.

Boutin, B. A. (2007)b: «De, que, pour, subordonnants et variation en français », Journées franco-allemandes de linguistique, organisées par F. Gadet et E. Guerin, Etudes de syntaxe : français parlé, français hors de France, créoles, 18 et 19 octobre 2007, Paris-X Nanterre, Actes en ligne, http://www.modyco.fr/?u s=8\&u a=810\&

Boutin, B. A. (2007)c: «Là, même, aussi, coordonnateurs d'attention», Colloque International PFC Regards croisés sur la phonologie du français contemporain, 6-8 décembre 2007, Maison des Sciences de l'Homme, Paris, http://www.projet-pfc.net/?u s=4\&u a=141\&.

Boutin, B. A. \& G. Turcsan (2006). «Groupes consonantiques dans le français ivoirien : variation, volatilité, vocalisation », Colloque international PFC : Phonologie du français : du social au cognitif, 7-9 décembre 2006, Maison des Sciences de l'Homme, Paris, http://www.projet-pfc.net/?u s=0\&u a=101\&.

Boutin, B. A., C. Lyche \& G. Prignitz (2007). «Les enquêtes PFC en Afrique », in S. Detey \& D. Nouveau (eds) : PFC-Enseignement du français \& Travaux en cours, Phonologie du Français Contemporain, Bulletin $n^{\circ} 7: 297-$ 330, http://www.projet-pfc.net/?u s=4\&u a=157\&.

Durand, J. \& C. Lyche (2008). French liaison in the light of corpus data. In B. Laks : Pour une phonologie de corpus. Journal of French Language Studies 18 : 33-66. Cambridge : CUP.

Durand, J., B. Laks \& C. Lyche (2002). La phonologie du français contemporain: usages, variétés et structure. C. Pusch \& W. Raible (eds.) Romanistische Korpuslinguistik- Korpora und gesprochene Sprache/Romance Corpus Linguistics - Corpora and Spoken Language. Tübingen: Gunter Narr Verlag, pp. 93-106.

Durand, J., B. Laks \& C. Lyche (2005). Un corpus numérisé pour la phonologie du français. G. Williams (ed.) $L a$ linguistique de corpus. Rennes: Presses Universitaires de Rennes. pp. 205-217. Actes du colloque 'La linguistique de corpus', Lorient, 12-14 septembre 2002.

Gadet, F. (2000). On n'en a pas fini avec les problèmes de recueil de corpus. Le français parlé. Actes du colloque international. Andersen, H. L \& A. B. Hansen (eds). Université de Copenhague, 29 au 30 octobre 1998. Université de Copenhague : Museum Tusculanum Press. Série : Etudes Romanes.

Gadet, F. (2007). La variation sociale en français. Paris : Ophrys.

Hattiger, J.-L. (1981). Morpho-syntaxe du groupe nominal dans un corpus de français populaire d'Abidjan. Thèse de $3^{\text {ème }}$ cycle. Université de Strasbourg.

Lafage, S. (2003 - 2004). Le lexique français de Côte d'Ivoire, appropriation et créativité. Tomes 1 et 2. Le français en Afrique, Revue du Réseau des Observatoires du Français Contemporain en Afrique, $\mathrm{n}^{\circ} 16$ \& 17. Paris : Didier - Erudition. 
Léon, P. (2005). Précis de phonostylistique. Parole et expressivité, Armand Colin, Coll. Fac. Linguistique.

Kouadio N'Guessan, J. (1992). Le nouchi abidjanais, naissance d'un argot ou mode linguistique passagère? In Des langues et des villes. Actes du Colloque International de Dakar, déc. 1990. Coll. Langues et développement, Paris : Didier Érudition.

Kouadio N'Guessan, J. (1999). Quelques traits morphosyntaxiques du français écrit en Côte d'Ivoire. Cahiers d'études et de recherches francophones, Langues, Vol. II, $\mathrm{n}^{\circ} 4: 301-314$, Paris : AUPELF - UREF

Kouadio N'Guessan, J. (2005). Le nouchi et les rapports dioula / français. In Des inventaires lexicaux du français en Afrique à la sociologie urbaine ... Hommage à Suzanne Lafage. Le français en Afrique, Revue du Réseau des Observatoires du Français Contemporain en Afrique n 19. Paris : Didier - Erudition.

Knutsen, A. M. (2007a). Variation du français à Abidjan (Côte d'Ivoire). Etude d'un continuum linguistique et social. Oslo, Université d'Oslo, Thèse de doctorat. Acta Humaniora.

Knutsen, A. M. (2007b). Le français à Abidjan (Côte d'Ivoire). Vers une analyse multidimensionnelle de la variation. Revue du Réseau des Observatoires du Français Contemporain en Afrique $\mathrm{n}^{\circ} 22$. Paris : Didier - Erudition, pp. 127-159.

Lyche, C. et al. (2007). PFC en terre africaine.

Milroy, L. (1980). Language and Social Networks. Oxford : Blackwell

Ploog, K. (2002). Le français à Abidjan. Pour une approche syntaxique du non-standard. Coll. Sciences du langage. Paris : CNRS Editions.

Prignitz, G. (2006b). Observations sur un corpus récent recueilli à Ouagadougou. In Le français en Afrique. Revue du Réseau des Observatoires du Français Contemporain en Afrique Noire ${ }^{\circ} 21$. Nice : I.L.F. / CNRS-UMR 6039, 193-210.

\footnotetext{
${ }^{1}$ Les entretiens semi directifs portaient sur les normes et les thèmes sont analysés en détail dans Boutin 2002 et 2003.

${ }^{2}$ L'enquête a eu lieu en 1999. De plus en plus, le nouchi perd son caractère cryptique et fusionne avec le français populaire pour devenir la langue identitaire ivoirienne hors d'un contexte demandant une langue soignée.

${ }^{3}$ Tous les renseignements sur les buts du projet, le protocole d'enquête, les points d'enquête, ainsi que les données obtenues sont disponibles sur le site Internet http://www.projet-pfc.net. Voir aussi Durand, Laks \& Lyche 2002, 2005 et, pour l'enquête en Afrique, Boutin, Lyche \& Prignitz 2007.
} 